Article

\title{
Effect of Simultaneous Exposure of Pigs to Streptococcus suis Serotypes 2 and 9 on Their Colonization and Transmission, and on Mortality
}

\author{
Niels Dekker ${ }^{1,2, *}$, Annemarie Bouma ${ }^{1}$, Ineke Daemen ${ }^{1}$, Hans Vernooij ${ }^{1}$ (D), Leo van Leengoed ${ }^{1}$, \\ Jaap A. Wagenaar ${ }^{2}$ and Arjan Stegeman ${ }^{1}$ \\ 1 Department of Farm Animal Health, Faculty of Veterinary Medicine, Utrecht University, \\ 3584 CL Utrecht, The Netherlands; A.Bouma@minez.nl (A.B.); A.J.J.M.Daemen@uu.nl (I.D.); \\ J.C.M.Vernooij@uu.nl (H.V.); L.vanLeengoed@uu.nl (L.v.L.); J.A.Stegeman@uu.nl (A.S.) \\ 2 Department of Infectious Diseases and Immunology, Faculty of Veterinary Medicine, Utrecht University, \\ 3584 CL Utrecht, The Netherlands; J.Wagenaar@uu.nl \\ * Correspondence: C.N.T.Dekker@uu.nl; Tel.: +31-030-253-6913
}

Received: 4 September 2017; Accepted: 26 September 2017; Published: 27 September 2017

\begin{abstract}
The distribution of Streptococcus suis serotypes isolated from clinically infected pigs differs between geographical areas, and varies over time. In several European countries, predomination of serotype 2 has changed to serotype 9 . We hypothesize a relation, with one serotype affecting the other in colonization and invasion. The aim of this study was to evaluate whether simultaneous exposure of pigs to serotypes 2 and 9 affects colonization and transmission of each type, and mortality. Thirty-six caesarean-derived/colostrum-deprived piglets were randomly assigned to three groups, and there housed pair-wise. At six weeks old, one pig per pair was inoculated with either one (serotype 2 or 9; mono-group) or two serotypes simultaneously (dual-group); the other pig was contact-exposed. Tonsillar and nasal samples were collected within three weeks post inoculation. Bacterial loads in samples were quantified using multiplex real-time polymerase chain reaction (PCR). Transmission rates of the serotypes among pigs were estimated using a mathematical Susceptible-Infectious (SI) model. Bacterial loads and transmission rates did not differ significantly between serotypes. Compared to the mono-group, in the dual-group the average serotype 2 load in tonsillar samples from contact pigs was reduced on days 1 to 4 and on day 6. Simultaneous exposure to the serotypes reduced the mortality hazard 6.3 times (95\% C.I.: 2.0-19.8) compared to exposure to serotype 2 only, and increased it 6.6 times ( $95 \%$ C.I.: 1.4-30.9) compared to exposure to serotype 9 only. This study indicates that serotype 2 load and mortality were affected in pigs exposed to these two serotypes.
\end{abstract}

Keywords: Streptococcus suis; serotype 2; serotype 9; pig; transmission; colonization; tonsil; nose; survival; qPCR

\section{Introduction}

Streptococcus suis (S. suis) is a pathogen for both pigs and humans, that is endemically present in commercial pig herds worldwide [1], and seems to be emerging in humans [2]. The natural habitat of $S$. suis in pigs is the upper respiratory tract (especially the tonsils and nasal cavities), as well as the gastrointestinal and genital tracts [1,3-5]. The respiratory route is generally thought to be the main route of horizontal transmission among pigs, and especially the tonsils are considered important reservoirs of S. suis [1,5]. According to the most accepted hypothesis, $S$. suis breaches the mucosal epithelium at either the upper respiratory tract or the gastrointestinal tract, and reaches the bloodstream, leading to systemic infection [3-5]. Although pigs of all ages may be colonized, severe systemic infections, characterised by meningitis, arthritis, endocarditis, pneumonia, and septicaemia, 
seem to occur mainly in young pigs (5-10 weeks of age) [1,3,5]. These clinical outbreaks result in considerable animal welfare problems and economic losses.

Based on differences in capsular polysaccharide (CPS) antigens, several serotypes of S. suis can be distinguished. So far, 33 serotypes of $S$. suis are known, although some are under debate as to whether they belong to the $S$. suis taxon, and new cps loci have been described in previously untypeable strains [6-8]. Often a pig is colonized by more than one serotype [9,10], but usually only one serotype is isolated from the internal organs of a pig that has suffered from a severe systemic infection [1]. The distribution of serotypes among clinical isolates differs between regions, and may also vary over time. This has been shown in many studies carried out in several regions/countries $[6,11]$. Results from some studies (performed in e.g., United Kingdom, Denmark, Spain, and the Netherlands) indicated that the predominant serotypes isolated from clinical cases have changed over the last decades [12-14]. In the Netherlands, for example, serotype 2 was found most often amongst isolates for several years (32\% serotype 2 versus $68 \%$ of a variety of serotypes), but from 1996 onwards, serotype 9 has been isolated more frequently (56-58\% type 9 versus $23-28 \%$ type 2) [15-17]. In Spain an even stronger increase in serotype 9 contribution was observed, i.e., from $4.4 \%$ or lower in the period 1991-1995 [16,18,19], to 54-65\% in the period 1998-2002 [6,14,20]. To develop sustainable control measures effective in reducing the incidence of $S$. suis infections, insight in the mechanism(s) underlying this change in serotype distribution among clinical isolates is needed.

The probability that a pig develops a clinical infection due to a certain serotype depends on the efficiency of that type to colonize the mucosa, its virulence or invasive capacity, and by the susceptibility of the pig. These characteristics may be affected by the presence of other serotypes of the same bacterial species, as has been shown for Streptococcus pneumoniae (S. pneumoniae) in humans [21-24], and in mouse models [25-28]. On farm level, this 'exposure history' depends on the relative rates at which various serotypes can spread among pigs.

The effect of exposure of pigs to more than one serotype on colonization of each of these and on the clinical outcome of an infection has been studied experimentally using pigs originating from a conventional farm [29]. In that study the presence of serotypes 2 and 9 in piglets from dams positively tested for both types was determined at the end of the lactation period at four weeks of age. However, as timing of exposure to either of the two serotypes was unknown in this study, its data cannot be used to quantify the relative transmission rates of serotypes amongst pigs.

Therefore, we carried out transmission experiments to study the transmission rates of serotypes 2 and 9 among piglets. The objective of this study was to evaluate whether S. suis serotypes 2 and 9, when inoculated simultaneously, would affect their respective capacity to colonize mucosa and to spread among pigs, and whether the clinical outcome of a systemic infection caused by one serotype was altered by the presence of the other. Results may help explain why a serotype shift from 2 to 9 has occurred.

\section{Results}

\subsection{Colonization}

Before inoculation all pigs tested S. suis negative by selective bacterial examination (SBE) and by multiplex real-time quantitative PCR (qPCR), within their limits of detection. All inoculated pigs tested positive at the first day after inoculation. All contact pigs became $S$. suis positive within two days post-exposure (DPE) to $S$. suis inoculated pigs. All positive pigs remained positive during the observational period.

In tonsillar samples, no significant differences in loads were observed at any of the sampling days when exposure to serotype 2 only was compared to exposure to serotype 9 only ( $p=0.99$ ). Tonsillar serotype 2 loads of inoculated pigs did not differ between the pigs exposed to one serotype (referred to as 'mono-group') and two serotypes (referred to as 'dual-group'); the mean loads in contact pigs in the dual-group were between 1.4 and 1.8 equivalent $\log _{10}$ colony forming units per sample 
$\left(\log _{10}\right.$ eq. CFU/sample) reduced on all first four days, and on day 6 after exposure. No differences were observed in tonsillar serotype 9 loads in the mono-group compared to the dual-group, except for day 1 after exposure. At that moment the dual-group showed a 1.6 and $1.7 \log _{10}$ eq. CFU/sample reduction in inoculated and contact pigs, respectively (Figure 1; Tables 1 and 2).

Table 1. Estimated differences in mean tonsillar serotype 2 loads (in $\log _{10}$ eq. CFU/sample) between pigs exposed to serotype 2 only ('Mono') and to both serotype 2 and 9 ('Dual') on different days post exposure ${ }^{1}$.

\begin{tabular}{|c|c|c|c|c|}
\hline \multirow[b]{2}{*}{ Time (DPE) ${ }^{1}$} & \multicolumn{2}{|l|}{ Inoculated Pigs } & \multicolumn{2}{|l|}{ Contact Exposed Pigs } \\
\hline & $\begin{array}{l}\text { Mean load ‘Mono' Minus Mean Load } \\
\text { 'Dual' ( } \log _{10} \text { eq. CFU/sample) }{ }^{2,3}\end{array}$ & $p$-Value & $\begin{array}{l}\text { Mean load 'Mono' Minus Mean Load } \\
\text { 'Dual' ( } \log _{10} \text { eq. CFU/sample) }{ }^{2,3}\end{array}$ & $p$-Value \\
\hline 1 & 0.64 & 0.352 & $\underline{1.57}$ & $\leq 0.001$ \\
\hline 2 & 0.46 & 0.873 & 1.40 & 0.004 \\
\hline 3 & 0.79 & 0.158 & $\underline{1.72}$ & $\overline{<0.001}$ \\
\hline 4 & 0.82 & 0.158 & 1.76 & $<0.001$ \\
\hline 5 & -0.06 & 0.999 & $\overline{0.87}$ & 0.351 \\
\hline 6 & 0.55 & 0.999 & $\underline{1.48}$ & $\underline{0.012}$ \\
\hline 7 & 0.31 & 0.999 & 1.24 & 0.078 \\
\hline 8 & -0.64 & 0.999 & 0.29 & 0.999 \\
\hline
\end{tabular}

${ }^{1}$ Data were analysed with a linear mixed regression model. In the final model time (expressed as 'day post exposure'; DPE), serotype exposure (mono or dual) and mode of infection (inoculated or contact exposed) were included as fixed effects, and pig as random effects; ${ }^{2}$ The difference in loads is a reflection of coefficient estimate $\beta$ of the final model. If significant, this difference and its $p$-value are underlined; ${ }^{3}$ The estimated mean load at $1 \mathrm{DPE}$ in dual group (i.e., the intercept of the final model) was $2.65 \log _{10}$ eq. CFU/sample and $0.80 \log _{10}$ eq. CFU/sample for the inoculated and contact pigs, respectively.

Table 2. Estimated differences in mean tonsillar serotype 9 loads (in $\log _{10}$ eq. CFU/sample) between pigs exposed to serotype 9 only ('Mono') and to both serotype 2 and 9 ('Dual') on different days post exposure ${ }^{1}$.

\begin{tabular}{|c|c|c|c|c|}
\hline \multirow[b]{2}{*}{ Time (DPE) ${ }^{1}$} & \multicolumn{2}{|l|}{ Inoculated Pigs } & \multicolumn{2}{|l|}{ Contact Exposed Pigs } \\
\hline & $\begin{array}{l}\text { Mean load 'Mono' Minus Mean Load } \\
\text { 'Dual' ( } \log _{10} \text { eq. CFU/sample) }{ }^{2,3}\end{array}$ & $p$-Value & $\begin{array}{l}\text { Mean load 'Mono' Minus Mean Load } \\
\text { 'Dual' ( } \log _{10} \text { eq. CFU/sample) } 2,3\end{array}$ & $p$-Value \\
\hline 1 & 1.61 & $\underline{0.004}$ & 1.72 & $\leq 0.001$ \\
\hline 2 & 0.86 & $\overline{0.361}$ & 0.96 & $\overline{0.240}$ \\
\hline 3 & 0.79 & 0.361 & 0.90 & 0.227 \\
\hline 4 & 0.29 & 0.999 & 0.40 & 0.999 \\
\hline 5 & 0.00 & 0.999 & 0.00 & 0.999 \\
\hline 6 & 0.00 & 0.999 & 0.09 & 0.999 \\
\hline 7 & 0.13 & 0.999 & 0.24 & 0.999 \\
\hline 8 & 0.03 & 0.999 & 0.13 & 0.999 \\
\hline
\end{tabular}

1,2 See legend Table $1 ;{ }^{3}$ The estimated mean load at 1 DPE in dual group (i.e., the intercept of the final model) was $1.46 \log _{10}$ eq. CFU/sample and $1.32 \log _{10}$ eq. CFU/sample for the inoculated and contact pigs, respectively.

Tonsillar serotype 2 loads in inoculated pigs of the mono-group did not differ from the loads in contact pigs, except for 2 DPE when inoculated pigs had on average $1.3 \log _{10}$ eq. CFU/sample higher loads than contact-infected pigs. In the dual-group the inoculated pigs showed 1.8 and 2.2 $\log _{10}$ eq. CFU/sample higher mean serotype 2 loads than their contact pigs on DPE 1 and 2, respectively. Mean serotype 9 loads did not differ between inoculated and contact pigs in any of the groups.

In nasal samples, mean serotype 9 loads did not differ between the mono- and dual-group on any of the days $(p>0.2)$ (Table 3). The mean nasal loads of serotype 2 did not differ between the monoand dual-group, except at 2 DPE when there was a reduction of on average $2.2 \log _{10}$ eq. CFU/sample $(p=0.01)$ in the contact pigs of the dual-group compared to the contact pigs of the mono-group (Figure 2; Table 4). 
Panel A: Serotype 2 in contact pigs

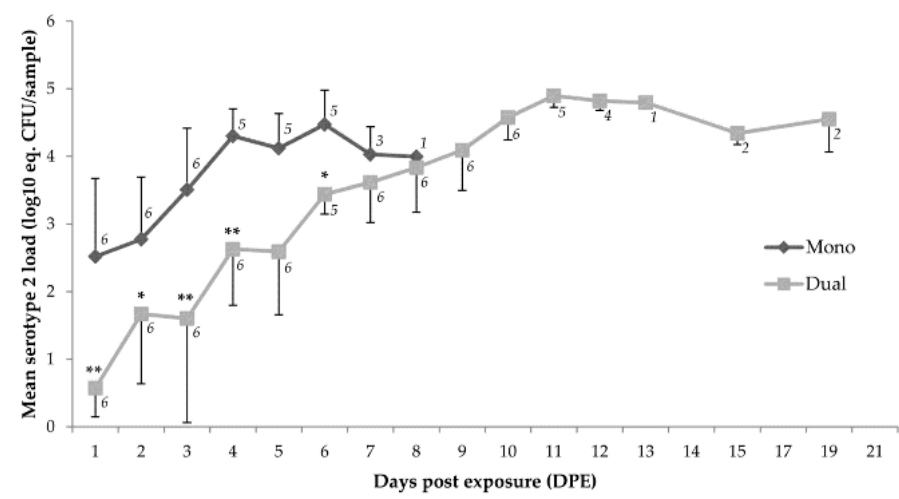

Panel C: Serotype 9 in contact pigs

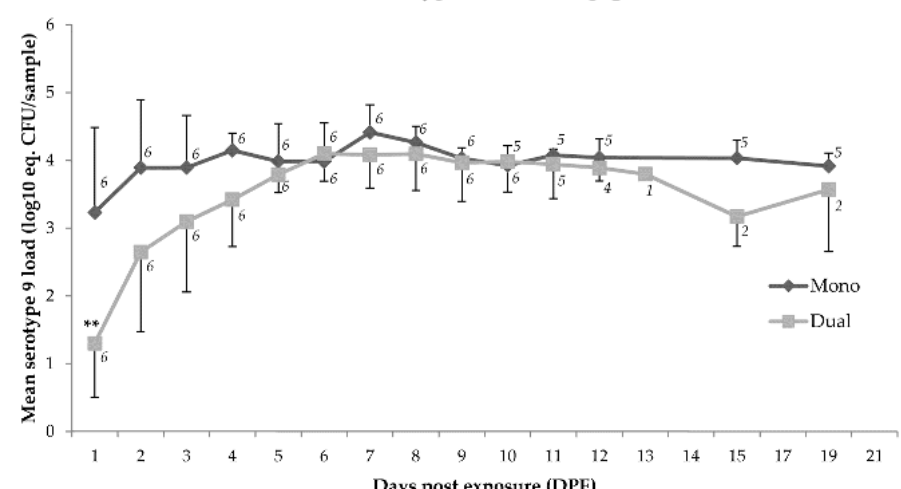

Panel B: Serotype 2 in inoculated pigs

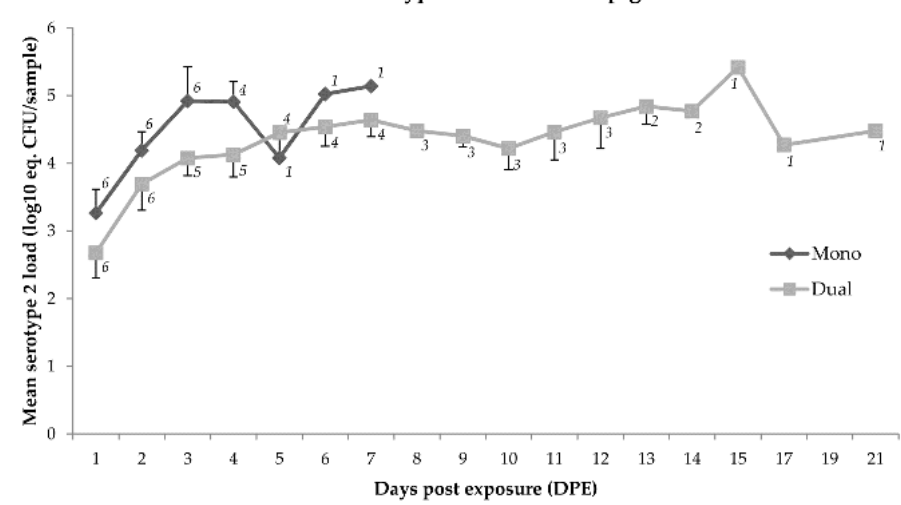

Panel D: Serotype 9 in inoculated pigs

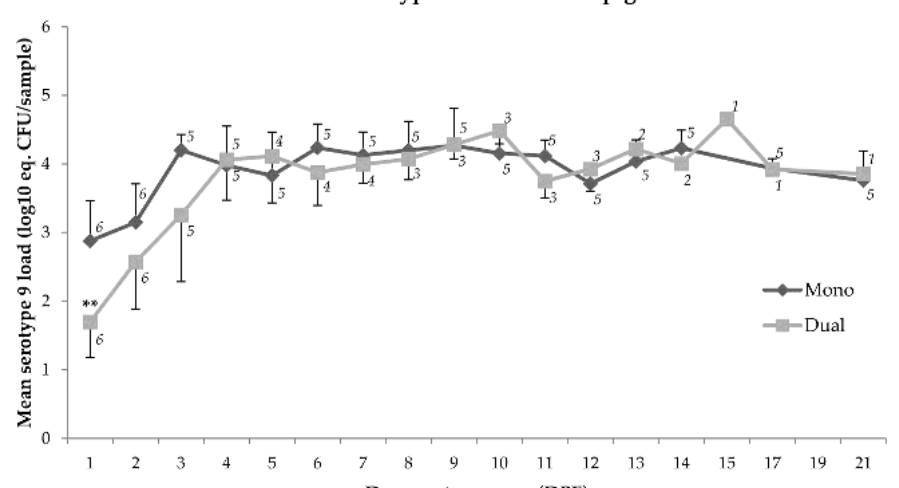

Days post exposure (DPE)

Figure 1. Mean loads of S. suis serotype 2 (panels A and B) or serotype 9 (panels $C$ and D) in tonsillar samples $\left(\log _{10}\right.$ eq. CFU/sample) per day post exposure (DPE) visualized for animal categories that differ in type of exposure (i.e., mono or dual), and in mode of infection (i.e., contact or inoculated). Significant differences in levels between mono- and dual-exposed pigs with the same infection mode are marked with $\left.{ }^{*}\right)$ if $p<0.05$ or $\left(^{* *}\right)$ if $p<0.01$. The bar at each data point reflects the standard deviation. Number of observations per data point is shown close to that point. 
Panel A: Serotype 2 in contact pig

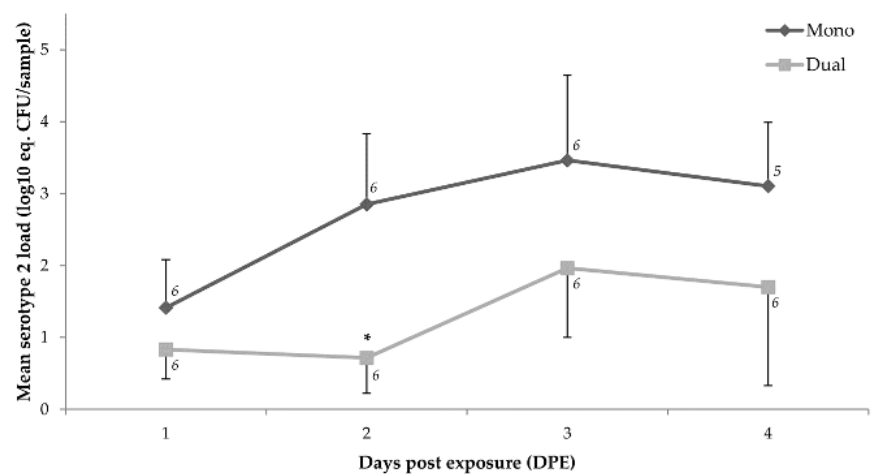

Panel C: Serotype 9 in contact pig

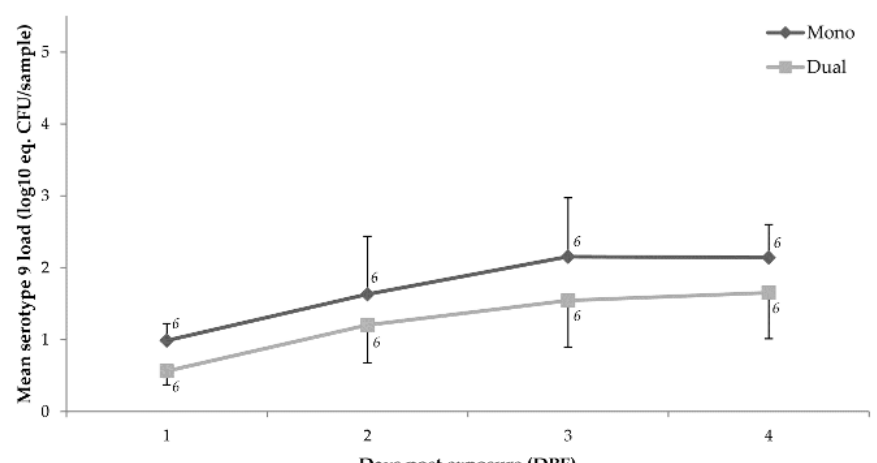

Panel B: Serotype 2 in inoculated pigs

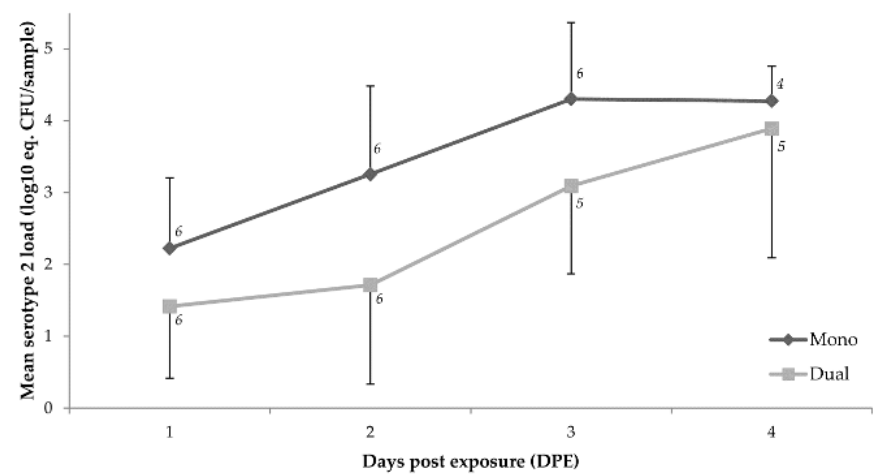

Panel D: Serotype 9 in inoculated pigs

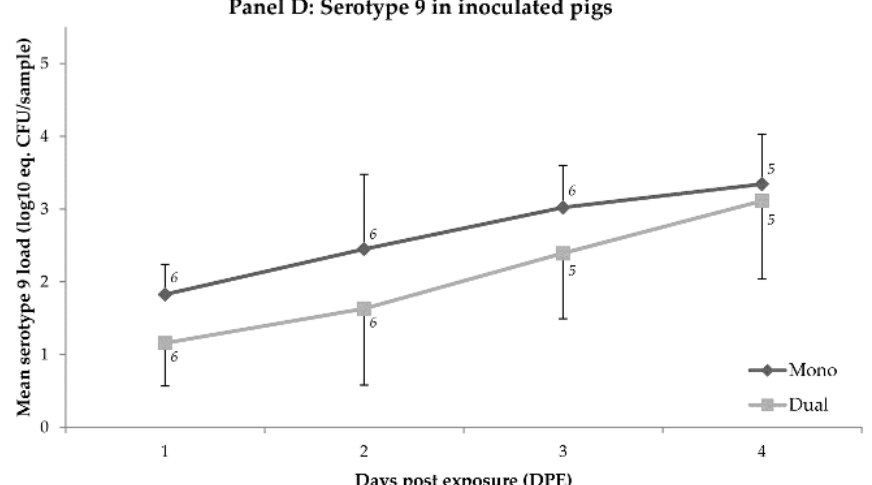

Figure 2. Mean load of S. suis serotype 2 (panels A and B) or serotype 9 (panels C and D) in nasal samples (log 10 eq. CFU/sample) per day post exposure (DPE) visualized for animal categories that differ in type of exposure (i.e., mono or dual), and in mode of infection (i.e., contact or inoculated). Significant differences in levels between mono- and dual-exposed pigs with the same infection mode are marked with $\left(^{*}\right)$ if $p<0.05$ or $\left(^{* *}\right)$ if $p<0.01$. The bar at each data point reflects the standard deviation. Number of observations per data point is shown close to that point. 
Table 3. Estimated differences in mean nasal serotype 9 loads (in $\log _{10}$ eq. CFU/sample) between pigs exposed to serotype 9 only ('Mono') and to both serotype 2 and 9 ('Dual') on different days post exposure ${ }^{1}$.

\begin{tabular}{|c|c|c|c|c|}
\hline & Inoculated Pigs & & Contact Exposed Pigs & \\
\hline Time (DPE) ${ }^{1}$ & $\begin{array}{c}\text { Mean load 'Mono' Minus Mean Load } \\
\text { 'Dual' ( } \log _{10} \text { eq. CFU/sample) } 2,3\end{array}$ & $p$-Value & $\begin{array}{c}\text { Mean load 'Mono' Minus Mean Load } \\
\text { 'Dual' ( } \log _{10} \text { eq. CFU/sample) } 2,3\end{array}$ & $p$-Value \\
\hline 1 & 0.94 & 0.233 & 0.67 & 0.709 \\
\hline 2 & 0.98 & 0.203 & 0.71 & 0.620 \\
\hline 3 & 0.72 & 0.650 & 0.45 & 0.999 \\
\hline 4 & 0.48 & 0.999 & 0.21 & 0.999 \\
\hline
\end{tabular}

1,2 See legend Table $1 ;{ }^{3}$ The estimated mean load at 1 DPE in dual group (i.e., the intercept of the final model) was $0.92 \log _{10}$ eq. CFU/sample and $0.28 \log _{10}$ eq. CFU/sample for the inoculated and contact pigs, respectively.

Table 4. Estimated differences in mean nasal serotype 2 loads (in $\log _{10}$ eq. CFU/sample) between pigs exposed to serotype 2 only ('Mono') and to both serotype 2 and 9 ('Dual') on different days post exposure ${ }^{1}$.

\begin{tabular}{|c|c|c|c|c|}
\hline \multirow[b]{2}{*}{ Time (DPE) ${ }^{1}$} & \multicolumn{2}{|l|}{ Inoculated Pigs } & \multicolumn{2}{|l|}{ Contact Exposed Pigs } \\
\hline & $\begin{array}{l}\text { Mean load 'Mono' Minus Mean Load } \\
\text { 'Dual' ( } \log _{10} \text { eq. CFU/sample) } 2,3\end{array}$ & $p$-Value & $\begin{array}{l}\text { Mean load 'Mono' Minus Mean Load } \\
\text { 'Dual' ( } \log _{10} \text { eq. CFU/sample) }{ }^{2,3}\end{array}$ & $p$-Value \\
\hline 1 & 0.73 & 0.999 & 1.29 & 0.352 \\
\hline 2 & 1.63 & 0.157 & 2.19 & $\underline{0.013}$ \\
\hline 3 & 1.15 & 0.566 & 1.70 & 0.133 \\
\hline 4 & 1.06 & 0.747 & 1.61 & 0.170 \\
\hline
\end{tabular}

1,2 See legend Table $1 ;{ }^{3}$ The estimated mean load at 1 DPE in dual group (i.e., the intercept of the final model) was $1.26 \log _{10}$ eq. CFU/sample and $0.34 \log _{10}$ eq. CFU/sample for the inoculated and contact pigs, respectively.

\subsection{Transmission}

All contact pigs in the mono-groups were colonized within one day after pairing with the inoculated pigs, and this applied to both serotypes. Within two days, all contact pigs in the dual-group were colonized with both types. For serotype 2, the transmission rates were estimated at 29.4/day (95\% C.I.: $0-\infty)$ in the mono-group, and 2.9/day (95\% C.I.: $1.2-6.9)$ in the dual-group $(p=0.99)$. The transmission rates for serotype 9 were $67 /$ day $(95 \%$ C.I.: $0-\infty)$ and $4.1 /$ day (95\% C.I.: $1.6-10.6)$, for the mono- and dual-group, respectively $(p=0.99)$.

\subsection{Clinical Disease and Mortality}

In all groups fever, lameness, and neurologic signs were observed. Fever was observed in all pigs exposed to serotype 2 only, in seven out of 12 exposed to serotype 9 only (six inoculated and one contact pig(s)), and in all except one contact pig of the dual-group. Lameness was observed in 10 pigs exposed to serotype 2 only (five inoculated, five contact), and the same was seen in the dual-group. In the group exposed to serotype 9 only, six inoculated pigs showed signs of lameness. Neurologic signs were seen in 10 pigs exposed to serotype 2 only (six inoculated, four contact), in five pigs exposed to serotype 9 only (four inoculated, one contact), and in seven pigs of the dual-group (four inoculated, three contact). High fever and sometimes neurologic signs were accompanied by vomiting in four pigs (two inoculated, two contact) of the group exposed to serotype 2 only, and in three pigs of the dual-group (two inoculated, one contact).

Twenty-three pigs died before the end of the experiment. Mortality occurred in all pigs exposed to serotype 2 only, in two exposed to serotype 9 only (one inoculated, one contact), and in nine pigs of the dual-group (five inoculated, four contact). Mortality occurred between 2-15 days after colonization in inoculated pigs, and between 3-13 days after colonization in contact pigs. Dual exposure reduced the hazard for mortality 6.3 times (95\% C.I.: 2.0-19.8) when compared to exposure to serotype 2 only, and increased mortality hazard 6.6 times (95\% C.I.: 1.4-30.9) when compared to exposure to serotype 9 only. The hazard for mortality was 41.6 times (95\% C.I.: 7.3-236.4) higher after exposure to serotype 
2 only compared to exposure to serotype 9 only (Figure 3). The hazard ratios did not differ significantly between inoculated and contact pigs.

In all fatal cases in the group exposed to serotype 2 only as well as in the dual-group, serotype 2 was isolated from internal organ samples of the pigs. In pigs from the group exposed to serotype 9 only, serotype 9 was isolated from internal organs.

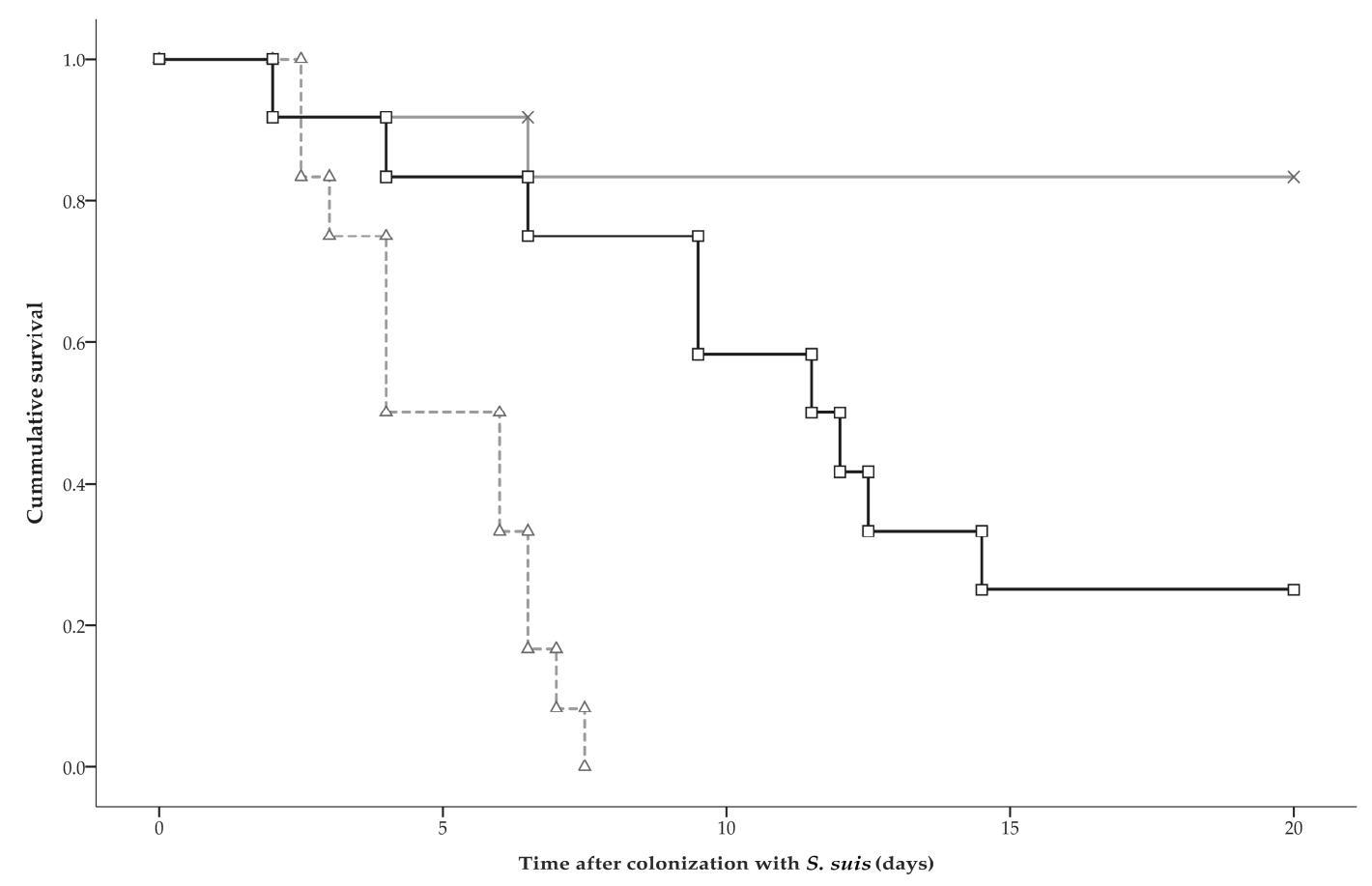

Figure 3. Kaplan-Meier survival curves for groups that were exposed to serotype 2 only (marked by $\Delta$ ), to serotype 9 only (marked by x), or to both serotypes (marked by $\square$ ). Survival analysis showed that the hazard for mortality in pigs exposed to both serotypes was 6.59 times (95\% C.I.: 1.41-30.86) increased compared to exposure to serotype 9 only, and 6.31 times (95\% C.I.: 2.01-19.77) reduced compared to exposure to serotype 2 only. The hazard ratios did not differ significantly between inoculated and contact pigs.

\section{Discussion}

The objective of this study was to examine whether inoculation of pigs with $S$. suis serotypes 2 and 9 simultaneously would affect the bacterial load in the oropharyngeal and nasal mucosa, the transmission of either serotype, and the mortality rate of colonized pigs. No significant differences were found in bacterial load or transmission rate of serotype 2 between pigs inoculated with serotype 2 only, or with both types. For serotype 9, a comparable result was found, except for the load on the first day after inoculation, which was approximately 40-fold lower in the pigs inoculated with both types, than in pigs inoculated with one type. In contact pigs exposed to both types, the serotype 2 load was 25 -fold to 60 -fold reduced for 5 days after colonization in comparison to pigs exposed to serotype 2 only; for serotype 9 a 40 -fold reduction in load in contact pigs of the dual-group in comparison to the mono-group was observed on the first day after exposure only. The transmission rates of both serotypes did not differ significantly, neither in the mono-group nor in the dual-group. Finally, simultaneous exposure to both serotypes reduced the mortality of contact pigs due to serotype 2 compared to mortality in contact pigs exposed to serotype 2 only. These findings suggest that 'natural' contact exposure to the two serotypes simultaneously affects the clinical outcome of an infection of one of these serotypes (i.e., serotype 2) in a population, possibly by affecting the mucosal load. The relative 
transmission rates were not affected in our study, suggesting that exposure history, i.e., the consecution at which strains colonize a pig, does not seem to be relevant.

In the pigs exposed to both types, serotype 2 load was more reduced compared to the pigs exposed to one type only, than serotype 9 was. These results suggest competitive interaction between the serotypes, directly (e.g., competition for space, nutrients) and/or indirectly (by e.g., interference of host factors and/or other locally present microbes), on adhesion, replication, and/or clearance of the bacteria. In an in vitro study with these serotypes, it was shown that serotype 9 was more resistant to killing/clearance by animal lysozyme than serotype 2 [30]. Other in vitro studies demonstrated that the biofilm-forming potential of serotype 9 was stronger than serotype 2 strains [31]. Biofilms enable evasion of host immune responses and more resistance against antimicrobials, facilitating persistence and dissemination of the bacteria [32]. Recently, Chuzeville et al. [33] studied in an in vitro setting with isogenic deficient mutants of $S$. suis, the effects of a bacterial protein known as antigen I/II (AgI/II), that was earlier linked to persistence of other streptococci in oral cavity [34], on processes linked to mucosal colonization ability/capacity. It was shown that AgI/II participated significantly in the aggregation of $S$. suis to a salivary glycoprotein, and contributed to biofilm formation, resistance to low $\mathrm{pH}$, as well as bacterial adhesion to extracellular matrix proteins and tracheal epithelial cells. Remarkably, these effects were all much stronger for serotype 9 than for serotype 2. For serotype 9 , the effect of AgI/II was also tested in an intranasal porcine model, and revealed the induction of higher mucosal loads; serotype 2 was, unfortunately, not included in that experiment [33]. Altogether, all these results obtained from only in vitro studies indicate a selective advantage of serotype 9 over serotype 2 in colonizing e.g., the upper respiratory tract.

In another study with these serotypes, it was found that cell constituents of serotype 9 induced a significantly higher activation level of the human Toll Like Receptor 2/6 complex than serotype 2, suggesting a stronger stimulation of the innate immune response at the mucosal level (e.g., induction of pro-inflammatory cytokines, recruitment of phagocytic cells [35]). These findings suggest interference of these mechanisms with bacterial growth, but do not explain the asymmetrical interference found in our experiment. Moreover, we did not observe a difference in bacterial load between serotype 2 and 9 in pigs exposed to one serotype only, suggesting that these mechanisms observed in vitro may not dominate or may not occur, in vivo. Whether these or other biological processes, suggested in other studies with S. suis [36,37] or other streptococci like S. pneumoniae (e.g., [38,39]), may play a role in interaction between serotypes 2 and 9 has not been determined in vivo either. The animal model presented here may offer a tool to study the effect of these processes on colonization and transmission of these S. suis serotypes. In addition, our model or its variants might also be of use in studying the interaction on $S$. suis colonization and transmission between other and/or more than two $S$. suis serotypes that often colonize pigs, between $S$. suis and other pig pathogens that seem to interfere with S. suis in vitro, like Actinobacillus pleuropneumoniae [40,41] or influenza virus [42], or even between different serotypes of human streptococci that are also able to colonize pigs, like S. pneumoniae [43].

Simultaneous exposure to serotypes 2 and 9 prolonged the survival time of contact pigs, due to a systemic serotype 2 infection in comparison to pigs exposed to serotype 2 only. This was not observed in inoculated pigs. Whether this is caused by the difference in total dose of $S$. suis exposure between mono-exposed $\left(1 \times 10^{9} \mathrm{CFU}\right)$ and dual-exposed $\left(2 \times 10^{9} \mathrm{CFU}\right)$ inoculated pigs, and/or between inoculated and 'more naturally' exposed contact pigs, is unknown and could be subject of further study. In contact pigs, the load of serotype 2 in the mono-group was significantly higher than in contacts in the dual-group. These differences were not observed in the inoculated pigs. These findings in more 'naturally' exposed pigs suggest a dose-dependent association between bacterial load and severity of clinical signs [44]. Reduction of bacterial load may be a way to reduce clinical problems if elimination of bacteria in a herd is not feasible. An important condition to evaluate such loads is the availability of a validated test to quantify the presence of (a) virulent strain(s); these are, however, not yet available for conventional pigs. 
Due to logistical and financial limits the number of pigs that could be included in the trial was restricted. As using homogenous groups increases the power of the study, we performed the experiment with caesarean derived, colostrum deprived (CDCD) pigs, to ensure the absence of interference due to serotypes or other micro-organisms already present, and passive maternal-specific immunity. With this study design, it is possible to detect differences in transmission if the reproduction ratio $(R)$ in one group is larger than one and in the other below one [45]. No differences in transmission were found, and both serotypes spread extensively $(R>1)$. However, we found differences in serotype 2 load between inoculated and contact pigs in the dual-group, suggesting a difference in infectiousness for serotype 2 . This difference may result in differences in transmission between the serotypes if longer transmission chains could be observed, e.g., in experiments where 'naturally', contact infected pigs have direct (same pen) or indirect (other pen in stable unit; 'airborne') contact with non-infected ones [45-47]. Moreover, small difference in transmission can also occur in the field, resulting in significant differences in the number and/or extent of outbreaks, as for example shown for pseudorabies virus [48,49]. If this also applies to $S$. suis, this may result in a shift in distribution of serotypes at the farm level. Small differences in spread cannot be found under experimental conditions, mainly because of the large number of animals required and the associated costs. The use of CDCD pigs and the controlled conditions makes extrapolation to the field more difficult. CDCD and conventional pigs differ in, amongst others, the composition of the mucosal flora and in passive immunity against S. suis, which may influence the course of disease [50]. Difference in the course of disease between $\mathrm{CDCD}$ and more conventional pigs after $S$. suis infection is suggested by the failure to induce clinical disease after intranasal inoculation with serotype 9 in Specific Pathogen Free (SPF) pigs, both with and without prior mucosal exposure to other pathogenic bacteria (Bordetella bronchiseptica) or acetic acid, in some previous experimental studies [44,51], but not in studies using (non-pre-treated) CDCD pigs ([47], this study).

Whether or not differences between CDCD and conventional pigs in, for example mucosal flora (e.g., in previous colonization with other S. suis strains), and in passive immunity interfere with colonization of a particular S. suis serotype is not known, and requires further field studies. Nevertheless, our study indicated that both serotypes differed in virulence, which is in accordance to earlier studies [44,51], and that virulent strains do not necessarily spread more extensively or rapidly than less virulent strains. Moreover, the presence of one serotype seems to affect the characteristics of the other, suggesting that replacement may occur. Whether this occurs in the field, and has contributed to the serotype shifts observed in practice [6], has to be determined.

\section{Materials and Methods}

\subsection{Inoculum}

S. suis serotype 2 strain 10 and S. suis serotype 9 strain 7997 were used as inocula (provided by H. Smith, Wageningen Bioveterinary Research (WBVR), Lelystad, the Netherlands). Both strains contain genes for suilysine (SLY) and muramidase-released protein (MRP), and strain 10 also has a gene encoding for extracellular factor (EF). Strain 10 belongs to MultiLocus Sequence Typing (MLST) clonal complex 1 (CC1), and strain 7997 to CC16.

An aliquot was taken from a $-80^{\circ} \mathrm{C}$ stock and cultured overnight on agar plates at $37^{\circ} \mathrm{C}$ and $5 \% \mathrm{CO}_{2}$. One colony was suspended in $10 \mathrm{~mL}$ Todd-Hewitt broth (TH) (BioTrading, Mijdrecht, the Netherlands), and incubated at $37^{\circ} \mathrm{C}$ for $3-4 \mathrm{~h}$ until an optical density of $0.5-0.6$ at $600 \mathrm{~nm}$ was reached. This suspension was stored overnight at $4{ }^{\circ} \mathrm{C}$, and was subsequently diluted tenfold in $\mathrm{TH}$ and cultured for $2 \mathrm{~h}$ at $37^{\circ} \mathrm{C}$ until an optical density of $0.5-0.6$ at $600 \mathrm{~nm}$. A total of $100 \mathrm{~mL}$ suspension was washed twice and suspended in $10 \mathrm{~mL}$ (for inoculation with one serotype) or $5 \mathrm{~mL}$ (for inoculation with both serotypes) physiologic saline solution. The bacterial concentration of the final suspension was for each strain $2-3 \times 10^{8} \mathrm{CFU}$ per $\mathrm{mL}$ for the pigs exposed to one serotype ('mono-group'), and $4-6 \times 10^{8} \mathrm{CFU} / \mathrm{mL}$ for the pigs exposed to both types ('dual-group'). 


\subsection{Pigs}

S. suis-free Landrace $x$ Yorkshire pigs were used. These pigs $(n=36)$ were obtained by caesarean section from sows from the farm of the Faculty of Veterinary Medicine, Utrecht, the Netherlands. The pigs were raised without colostrum. They were first fed with milk replacers and after 2-3 weeks of age there was a transitional period to gamma-irradiated pelleted concentrates (Trouw Nutrition, Amersfoort, The Netherlands). Probiotic bacteria Enterococcus faecium (M74 ${ }^{\circledR}$, Chr. Hansen, Hørsholm, Denmark), Bacillus licheniformis (DSM 5749), and Bacillus subtilis (DSM 5750) were added to the feed. Until the start of the experiment the pigs were successively housed in groups of 4-5 animals in isolators (weeks 0 to 4 ) and altogether in one ground floor pen (weeks 5 and 6).

\subsection{Experimental Design}

The experiment was performed in three separated stable units in one building. All stable units had positive air pressure conditions. Each unit had its own ventilation system and hygiene barrier (including shower and airlock). Within each unit, 12 pigs were housed pair-wise in boxes that were separated by a distance of $1.0 \mathrm{~m}$ (Figure 4). The boxes had closed walls (height: $80 \mathrm{~cm}$ ) and a plasticized iron grid floor with rubber lying area, and contained a feeding trough, drinking nipple, and bite sticks. The total ground area was $1.2 \mathrm{~m}^{2}$ per box.

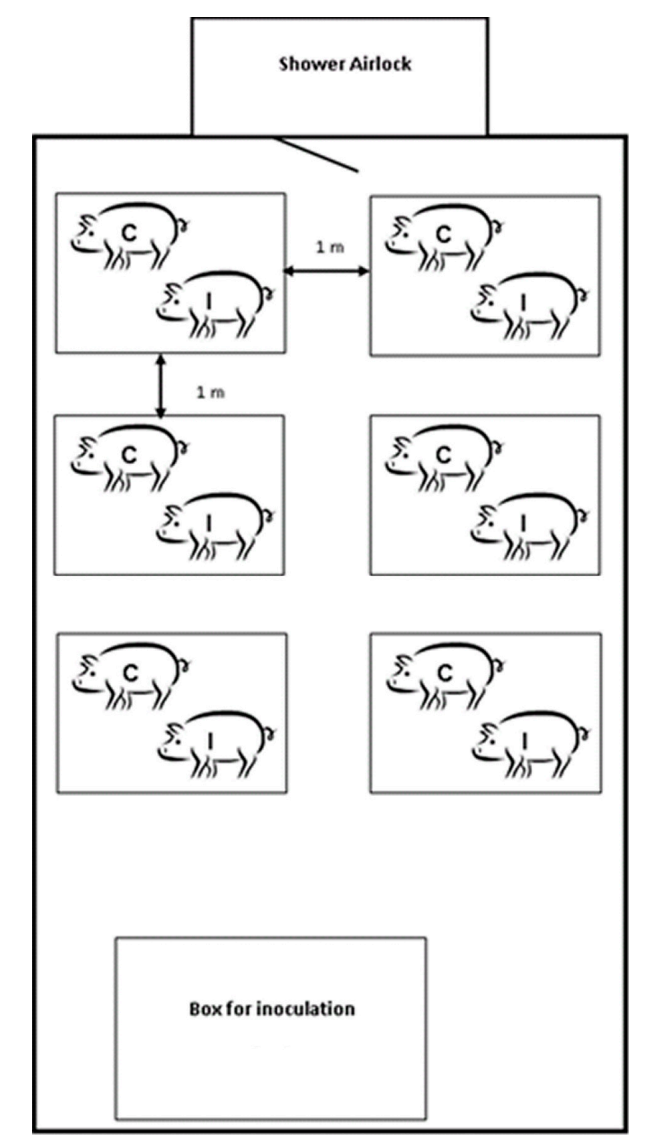

Figure 4. Design of one stable unit as used in the animal experiment. In the unit, each of the six small boxes contained one pig inoculated with $S$. suis (I) and one contact exposed pig (C). The largest box was only used to house the inoculated pigs during inoculation and the following $48 \mathrm{~h}$. In the study, three similar stable units were used. Within a unit, all inoculated pigs were either inoculated with serotype 2 (Group 1), or with serotype 9 (Group 2), or with both serotypes (Group 3). 
Pigs in Unit 1 were exposed to serotype 2 (Group 1), in Unit 2 to serotype 9 (Group 2), and in Unit 3 to both serotypes 2 and 9 (Group 3). To start the infection chain, one pig in each pair was intranasally inoculated with $5 \mathrm{~mL}$ saline containing $1 \times 10^{9} \mathrm{CFU}$ S. suis serotype 2 (Group 1), $1 \times 10^{9} \mathrm{CFU}$ S. suis serotype 9 (Group 2), or both serotype 2 and 9 in doses of $1 \times 10^{9} \mathrm{CFU}$ each $\left(2 \times 10^{9} \mathrm{CFU}\right.$ S. suis in total) (Group 3) [52]. Inoculation was performed in a ground pen located at a distance of more than $1.5 \mathrm{~m}$ from the nearest boxes (Figure 4) and at two days before the entrance of the non-inoculated, contact exposed pigs (contact pigs) in that unit to prevent infection of these contact pigs by the inoculum directly. Two days post inoculation (DPI), the inoculated pigs and the contact pigs were moved to the boxes to form pairs. The pigs were then six weeks old. Pigs were randomly assigned over the experimental groups (Groups 1, 2 and 3), pairs, and modes of infection (inoculated or contact). To prevent infections by animal handling, animal handlers showered and changed clothes before unit entrance, and wore face masks, sterile gloves, and hair covers. Coveralls, gloves, and overshoes were changed before treatment of each box. The experiments were approved by the Animal Care and Ethics Committee of Utrecht University, in accordance with the Dutch law on experimental animals (approval number DEC 2011.II.08.125).

\subsection{Clinical Inspection}

Pigs were inspected daily at $8.00 \mathrm{~h}$ and $19.00 \mathrm{~h}$, and clinical observations and rectal temperatures were recorded. Diseased pigs were treated with fentanyl $(10 \mu \mathrm{g} / \mathrm{kg}$ body weight), and severely affected pigs were euthanized for ethical reasons. Remaining pigs were sacrificed at the end of the experiment, which was at 21 DPI (similar to 21 DPE for inoculated and 19 DPE for contact pigs).

\subsection{Sampling}

Tonsillar brushing samples were taken in all pigs at similar time points, starting at five days and one day before inoculation. After inoculation for inoculated pigs samples were taken daily at 1-14 and 17 DPE, and at the experiments end (21 DPE). For the contact pigs this was at 1-12, 15 and 19 DPE. Per box, the contact pig was sampled before the inoculated pig. To obtain a tonsillar brushing sample the mouth was opened by a sterile steel wedge, and both palatine tonsillar areas were brushed for $3 \mathrm{~s}$ each with a sterile toothbrush. Nasal samples were taken at 1-4 DPE for both inoculated and contact pigs by rotating a swab (type MW102, MedicalWire and Equipment, Corsham, UK) in both nostrils for $2 \mathrm{~s}$ each. The brush and swab heads were cut off with sterile wire-cutters, put in separate sterile tubes containing $10 \mathrm{~mL}$ (tonsil) or $1.3 \mathrm{~mL}$ (nose) saline, and transported to the laboratory immediately. In the lab the tubes were thoroughly mixed, and $1 \mathrm{~mL}$ (tonsil) or $0.5 \mathrm{~mL}$ (nose) of each sample suspension was transferred into a vial. The vials were stored at $-20^{\circ} \mathrm{C}$ until further processing. On all pigs post-mortem examination was performed. Macroscopically affected organs were sampled for bacterial examination.

\subsection{Laboratory Tests}

\subsubsection{Bacterial Examination}

To test the $S$. suis status of the pigs before the experiment, the samples taken before inoculation were submitted to PCR (see below) and to selective bacterial examination (SBE) [52]. For SBE, briefly, 10-fold serial sample dilutions $\left(10^{1}\right.$ to $\left.10^{4}\right)$ were made, and $50 \mu \mathrm{L}$ of the undiluted sample and each dilution was plated on a selective agar plate containing Columbia agar, $6 \%$ sheep blood, $0.2 \mu \mathrm{g} / \mathrm{mL}$ crystal violet and colistin/ oxolinic acid (BioTrading) [52,53]. The culture plates were incubated at $37^{\circ} \mathrm{C}$ and $5 \% \mathrm{CO}_{2}$. Colonies that were suspected to be $\mathrm{S}$. suis on the basis of colony morphology were subcultured and tested for amylase activity [54]. Isolates that showed amylase activity were further tested with API 20 Strep (bioMérieux, Marcy l'Etoile, France), and serotyped by slide agglutination with S. suis serotype specific antisera (serotype 1, 2 and 9) (WBVR) and PCR (see below). 
Each sample taken from a lesion seen at necropsy was plated on two Columbia agar plates containing $6 \%$ sheep blood and one MacConkey agar plate (Biotrading). All three plates were incubated for $18-24 \mathrm{~h}$ at $37^{\circ} \mathrm{C}$; one Columbia agar plate at $5 \% \mathrm{CO}_{2}$, and the other plates at a normal atmosphere. Colonies that were suspected to be S. suis based on colony morphology were selected (in total 10 per animal), subcultured, tested for amylase-activity and serotyped by slide agglutination (see above) and by PCR (see below).

All amylase-positive isolates were suspended in $0.5 \mathrm{~mL}$ Tris-HCl EDTA (TE) buffer pH 7.5 (10 mM) and stored at $-20{ }^{\circ} \mathrm{C}$ until DNA isolation and PCR was performed.

\subsubsection{Multiplex Real-Time Quantitative PCR}

S. suis was detected and quantified using a multiplex qPCR on all nasal and tonsillar samples, as described before [55]. Briefly, after thawing, the samples were mixed and centrifuged $(13,000 \mathrm{G}$, $5 \mathrm{~min}$ ) in succession, and the supernatant was discarded with a pipette. Subsequently, $200 \mu \mathrm{L}$ InstaGene ${ }^{\mathrm{TM}}$ Matrix (Bio-Rad, Hercules, CA, USA) was added to each sample, as well as $5 \mu \mathrm{L}$ DNA containing 10 copies of pUC19 as internal control to monitor PCR replication efficiency. DNA was isolated according to the manufacturer instructions of InstaGene ${ }^{\mathrm{TM}}$ Matrix (Bio-Rad), with some minor modifications that were recently published [56].

The PCR targeted on the cps9H gene of S. suis serotype 9, the cps2J gene of serotype 2 (and 1/2), and on pUC19 (the internal control) by using primers and probes that were described before $[52,55,57]$. The primers/probe-mix consisting of $300 \mathrm{nM}$ of each cps2J- and cps9H-primer, $125 \mathrm{nM}$ cps9H-probe, $150 \mathrm{nM}$ cps2J-probe, and $100 \mathrm{nM}$ of each pUC19 primer and probe. The PCR mixture $(20 \mu \mathrm{L})$ contained $10 \mu \mathrm{L}$ LC480 Probes Master ( $2 \times)$ (Roche Diagnostics, Woerden, the Netherlands), $1 \mu \mathrm{L}$ of the primers/probe mix, $4 \mu \mathrm{L}$ PCR-grade water (Roche Diagnostics) and $5 \mu \mathrm{L}$ template DNA. DNA-amplification was carried out in a LightCycler ${ }^{\circledR} 480-$ II Instrument (Roche Diagnostics GmbH, Mannheim, Germany). The PCR conditions were as follows: $95^{\circ} \mathrm{C}$ for $10 \mathrm{~min}, 45$ cycles of $95^{\circ} \mathrm{C}$ for $10 \mathrm{~s}, 58^{\circ} \mathrm{C}$ for $30 \mathrm{~s}$, and $72{ }^{\circ} \mathrm{C}$ for $30 \mathrm{~s}$, and a final extension at $72{ }^{\circ} \mathrm{C}$. The loads of each serotype in a sample were calculated using standard curves and the LightCycler ${ }^{\circledR} 480$ algorithm, as described [55]. The minimal detection limits of the qPCR were similar for both serotypes, i.e., 1 eq. CFU per sample in PCR reaction (further designated as 'eq. CFU/sample') [55]. Loads higher than the highest concentration in the standard curve (i.e., $1 \times 10^{4}$ eq. CFU/sample) were extrapolated using the algorithm of the LightCycler ${ }^{\circledR} 480$ Instrument.

Samples were retested if the crossing point of the pUC19 reaction exceeded the previously determined cut-off value of either tonsillar or nasal samples, and excluded from analysis if retesting led to the same result. In our experiment no tonsillar or nasal samples had to be excluded. Before analysis, the loads were $\log _{10}$ transformed $\left(\log _{10} \mathrm{CFU}\right)$ for data normalization.

\subsection{Statistical Analysis}

\subsubsection{S. suis Colonization}

Colonization data were used to compare loads of either serotype 2 or 9 after exposure to one serotype, and to evaluate whether a dual infection affects the loads of either of the types. Data of all tonsillar samples were analysed in a linear mixed effects model procedure with the assumption of a normal distribution for the outcome. The load of S. suis ( $\log _{10}$ eq. CFU/sample), either serotype 2 or 9 , was used as dependent variable.

Three different models were made; two with data on one serotype ( 2 or 9$)$ to evaluate the effect of exposure to two types on colonization of a single serotype, and one model with data on both serotypes from the mono-group to compare the colonization capacity between the two serotypes.

The models had a random effect for pig to correct for repeated measurements within the same animal. Time (DPE) and serotype(s) exposure type (mono or dual) were included as explanatory variables. Mode of infection (inoculated or contact) and interactions between the above-mentioned 
factors were tested by backward selection procedure using the Akaike's Information Criteria (AIC) to select the best model [58]. The assumptions of normality of residuals and equal variances of the final models were confirmed by visual inspection of quantile-quantile plots, plots of standardized residuals against predicted values, and plots of standardized residuals against all predictor variables. Based on this assumptions check, in the serotype 9 model differences in variances dependent on time (DPE), and for serotype 2 also on mode of infection (inoculated or contact), had to be corrected by adding a variance structure allowing different levels of variances.

This whole modeling procedure was also performed for comparison of serotype 2 and 9 loads between groups exposed to a single serotype, except that 'exposure type' (mono or dual) was replaced by 'serotype exposure' ( 2 or 9 ) as fixed factor. The same procedure was applied for loads in nasal samples, but no correction for variance structure was needed.

All models were fitted using the statistical program R (version 2.13.0) (R Foundation for Statistical Computing, Vienna, Austria) with use of the LME function from the NLME-library [59].

On the $p$-values of the coefficients' estimates of the final model, the Benjamini \& Yekutieli post-hoc procedure was applied to correct for multiple comparisons. Differences in outcome variables were considered significant if $p<0.05$.

\subsubsection{Animal Survival}

Cox proportional hazard ( $\mathrm{CPH})$ analysis was performed to evaluate the effect of exposure to both types in comparison to one, and of exposure to serotype 2 only compared to serotype 9 only on animal survival. Time was expressed as day after the first positive nasal and/or tonsillar sample in that pig. In all inoculated pigs this was at $1 \mathrm{DPE}$, in contact pigs this varied. The $\mathrm{CPH}$ model assumes that there is a basal hazard ratio that can be increased or decreased due to factors included in the model. We first added 'serotype exposure' (serotype 2 or 9, or both types) and 'mode of infection' (inoculated or contact) and their interaction as fixed factors to the model, and frailty to take repeated measurements within one box into account. After backward selection based on AIC values, the interaction was excluded. Compliance with the proportional hazard assumption was tested graphically by plotting the Schoenfeld residuals against time, and by performing a Cox.zph test. The Benjamini \& Yekutieli post-hoc procedure was applied to correct for multiple comparisons. Differences in hazard ratios were considered significant if $p<0.05$.

\subsubsection{Transmission}

To evaluate the effect of exposure to both S. suis serotype 2 and 9 on the spread of each of these types within their host population, and to compare the rate of $S$. suis transmission between hosts exposed to serotype 2 or 9 , transmission was quantified per serotype by using a stochastic Susceptible-Infectious (SI) model [60]. With this model, the transmission rate $\beta$ can be estimated, which is defined as the average number of secondary infections caused by one typical infectious individual in a susceptible population per unit of time $[60,61]$. For estimation of $\beta$ we used an approach described previously [52,62], with small modifications. Pigs were classified as being infectious (I) for a particular S. suis serotype when tonsillar and/or nose samples were tested positive for that serotype. The effect of exposure to both types, or of exposure to a particular serotype only on transmission parameter $\beta$ was tested using a generalized linear model with a complementary-Log-Log link function and $\log \mathrm{I} / \mathrm{N}$ as offset variable $[60,62,63]$. For evaluation of the effect of exposure to both types, the null hypothesis was that it would have no effect on transmission rate, irrespective of the serotype studied. For comparison of serotypes 2 and 9, the null hypothesis was that both the serotypes would have a similar transmission rate, after simultaneous exposure to both types as well as exposure to one type. The Benjamini \& Yekutieli post-hoc procedure was applied to correct for multiple comparisons. Differences were considered significant if $p<0.05$. Power calculation showed that to detect a difference in transmission between reproduction ratio $R>10$, as estimated before for $S$. suis serotype 9 [52], and a situation where spread is blocked by intervention $(R<1)$, at least 60 pigs were needed in 
this experimental set up [45,61]. For logistic and methodological reasons we decided to perform the study in two separate experimental rounds. However, based on the unexpected high disease severity and considering the transmission results in the first round (i.e., absence of transmission reduction; homogeneity in pairs) we decided to cancel a second trial for reasons of animal welfare. Statistical analysis of both animal survival and transmission was performed in R (version 2.13.0).

Acknowledgments: The authors wish to thank Linda Peeters for her technical assistance, and the animal care personnel for their assistance in sampling the pigs. This study was financially supported by Utrecht University, The Netherlands.

Author Contributions: N.D., A.B., L.L., J.A.W. and A.S. conceived and designed the experiments; N.D., A.B., I.D. and L.L. performed the experiments; N.D., H.V. and A.S. analyzed the data; A.S. contributed reagents/materials/analysis tools; N.D., A.B., H.V., L.L., J.A.W. and A.S. wrote the paper.

Conflicts of Interest: The authors declare no conflict of interest.

\section{References}

1. Gottschalk, M. Streptococcosis. In Diseases of Swine, 10th ed.; Zimmerman, J., Karriker, L., Ramirez, A., Schwarz, K., Stevenson, G., Eds.; Wiley-Blackwell: West Sussex, UK, 2012; pp. 841-855.

2. Gottschalk, M.; Xu, J.; Calzas, C.; Segura, M. Streptococcus suis: A new emerging or an old neglected zoonotic pathogen? Future Microbiol. 2010, 5, 371-391. [CrossRef] [PubMed]

3. Fittipaldi, N.; Segura, M.; Grenier, D.; Gottschalk, M. Virulence factors involved in the pathogenesis of the infection caused by the swine pathogen and zoonotic agent Streptococcus suis. Future Microbiol. 2012, 7, 259-279. [CrossRef] [PubMed]

4. Segura, M.; Fittipaldi, N.; Calzas, C.; Gottschalk, M. Critical Streptococcus suis Virulence Factors: Are They All Really Critical? Trends Microbiol. 2017, 25, 585-599. [CrossRef] [PubMed]

5. Segura, M.; Calzas, C.; Grenier, D.; Gottschalk, M. Initial steps of the pathogenesis of the infection caused by Streptococcus suis: Fighting against nonspecific defenses. FEBS Lett. 2016, 590, 3772-3799. [CrossRef] [PubMed]

6. Goyette-Desjardins, G.; Auger, J.P.; Xu, J.; Segura, M.; Gottschalk, M. Streptococcus suis, an important pig pathogen and emerging zoonotic agent-An update on the worldwide distribution based on serotyping and sequence typing. Emerg. Microbes Infect. 2014, 3, e45. [CrossRef] [PubMed]

7. Zheng, H.; Ji, S.; Liu, Z.; Lan, R.; Huang, Y.; Bai, X.; Gottschalk, M.; Xu, J. Eight Novel Capsular Polysaccharide Synthesis Gene Loci Identified in Nontypeable Streptococcus suis Isolates. Appl. Environ. Microbiol. 2015, 81, 4111-4119. [CrossRef] [PubMed]

8. Pan, Z.; Ma, J.; Dong, W.; Song, W.; Wang, K.; Lu, C.; Yao, H. Novel variant serotype of Streptococcus suis isolated from piglets with meningitis. Appl. Environ. Microbiol. 2015, 81, 976-985. [CrossRef] [PubMed]

9. Flores, J.L.; Higgins, R.; D’Allaire, S.; Charette, R.; Boudreau, M.; Gottschalk, M. Distribution of the different capsular types of Streptococcus suis in nineteen swine nurseries. Can. Vet. J. 1993, 34, 170-171. [PubMed]

10. Wisselink, H.J.; Joosten, J.J.; Smith, H.E. Multiplex PCR assays for simultaneous detection of six major serotypes and two virulence-associated phenotypes of Streptococcus suis in tonsillar specimens from pigs. J. Clin. Microbiol. 2002, 40, 2922-2929. [CrossRef] [PubMed]

11. Gottschalk, M.; Lacouture, S. Canada: Distribution of Streptococcus suis (from 2012 to 2014) and Actinobacillus pleuropneumoniae (from 2011 to 2014) serotypes isolated from diseased pigs. Can. Vet. J. 2015, 56, 1093-1094. [PubMed]

12. Heath, P.J.; Hunt, B.W. Streptococcus suis serotypes 3 to 28 associated with disease in pigs. Vet. Rec. 2001, 148, 207-208. [CrossRef] [PubMed]

13. Aarestrup, F.M.; Jorsal, S.E.; Jensen, N.E. Serological characterization and antimicrobial susceptibility of Streptococcus suis isolates from diagnostic samples in Denmark during 1995 and 1996. Vet. Microbiol. 1998, 60, 59-66. [CrossRef]

14. Tarradas, C.; Perea, A.; Vela, A.I.; Goyache, J.; Dominguez, L.; Fernández-Garaizabal, J.F.; Borge, C.; Huerta, B.; Luque, I. Distribution of serotypes of Streptococcus suis isolated from diseased pigs in Spain. Vet. Rec. 2004, 154, 665-666. [CrossRef] [PubMed] 
15. Jacobs, A.A.; van den Berg, A.J.; Baars, J.C.; Nielsen, B.; Johannsen, L.W. Production of suilysin, the thiolactivated haemolysin of Streptococcus suis, by field isolates from diseased pigs. Vet. Rec. 1995, 137, $295-296$. [CrossRef] [PubMed]

16. Wisselink, H.J.; Smith, H.E.; Stockhofe-Zurwieden, N.; Peperkamp, K.; Vecht, U. Distribution of capsular types and production of muramidase-released protein (MRP) and extracellular factor (EF) of Streptococcus suis strains isolated from diseased pigs in seven European countries. Vet. Microbiol. 2000, 74, 237-248. [CrossRef]

17. Schultsz, C.; Jansen, E.; Keijzers, W.; Rothkamp, A.; Duim, B.; Wagenaar, J.A.; van der Ende, A. Differences in the population structure of invasive Streptococcus suis strains isolated from pigs and from humans in the Netherlands. PLoS ONE 2012, 7, e33854. [CrossRef] [PubMed]

18. Luque, I.; Tarradas, C.; Arenas, A.; Maldonado, A.; Astorga, R.; Perea, A. Streptococcus suis serotypes associated with different disease conditions in pigs. Vet. Rec. 1998, 142, 726-727. [CrossRef] [PubMed]

19. Prieto, C.; Pena, J.; Suarez, P.; Imaz, M.; Castro, J.M. Isolation and distribution of Streptococcus suis capsular types from diseased pigs in Spain. Zentralbl. Veterinarmed. B 1993, 40, 544-548. [CrossRef] [PubMed]

20. Vela, A.I.; Goyache, J.; Tarradas, C.; Luque, I.; Mateos, A.; Moreno, M.A.; Borge, C.; Perea, J.A.; Domínguez, L.; Fernández-Garayzábal, J.F. Analysis of genetic diversity of Streptococcus suis clinical isolates from pigs in Spain by pulsed-field gel electrophoresis. J. Clin. Microbiol. 2003, 41, 2498-2502. [CrossRef] [PubMed]

21. Auranen, K.; Mehtälä, J.; Tanskanen, A.S.; Kaltoft, M. Between-strain competition in acquisition and clearance of pneumococcal carriage-Epidemiologic evidence from a longitudinal study of day-care children. Am. J. Epidemiol. 2010, 171, 169-176. [CrossRef] [PubMed]

22. Erästö, P.; Hoti, F.; Granat, S.M.; Mia, Z.; Mäkelä, P.H.; Auranen, K. Modelling multi-type transmission of pneumococcal carriage in Bangladeshi families. Epidemiol. Infect. 2010, 138, 861-872. [CrossRef] [PubMed]

23. Brugger, S.D.; Frey, P.; Aebi, S.; Hinds, J.; Mühlemann, K. Multiple colonization with S. pneumoniae before and after introduction of the seven-valent conjugated pneumococcal polysaccharide vaccine. PLoS ONE 2010, 5, e11638. [CrossRef] [PubMed]

24. Lipsitch, M.; Abdullahi, O.; D’Amour, O.A.; Xie, W.; Weinberger, D.M.; Tchetgen Tchetgen, E.; Scott, J.A.G. Estimating rates of carriage acquisition and clearance and competitive ability for pneumococcal serotypes in Kenya with a Markov transition model. Epidemiology 2012, 23, 510-519. [CrossRef] [PubMed]

25. Lipsitch, M.; Dykes, J.K.; Johnson, S.E.; Ades, E.W.; King, J.; Briles, D.E.; Carlone, G.M. Competition among Streptococcus pneumoniae for intranasal colonization in a mouse model. Vaccine 2000, 18, 2895-2901. [CrossRef]

26. Marks, L.R.; Reddinger, R.M.; Hakansson, A.P. High levels of genetic recombination during nasopharyngeal carriage and biofilm formation in Streptococcus pneumoniae. MBio 2012, 3, e00200-e00212. [CrossRef] [PubMed]

27. Dawid, S.; Roche, A.M.; Weiser, J.N. The blp bacteriocins of Streptococcus pneumoniae mediate intraspecies competition both in vitro and in vivo. Infect. Immun. 2007, 75, 443-451. [CrossRef] [PubMed]

28. Trzciński, K.; Li, Y.; Weinberger, D.M.; Thompson, C.M.; Cordy, D.; Bessolo, A.; Malley, R.; Lipsitch, M. Effect of serotype on pneumococcal competition in a mouse colonization model. MBio 2015, 6, e00902-e00915. [CrossRef] [PubMed]

29. Swildens, B. Detection and Transmission of Extracellular Factor Producing Streptococcus suis Serotype 2 Strains in Pigs. Ph.D. Thesis, Utrecht University, Utrecht, The Netherlands, 17 November 2009.

30. Wichgers Schreur, P.J.; van Weeghel, C.; Rebel, J.M.; Smits, M.A.; van Putten, J.P.; Smith, H.E. Lysozyme resistance in Streptococcus suis is highly variable and multifactorial. PLoS ONE 2012, 7, e36281. [CrossRef] [PubMed]

31. Dawei, G.; Liping, W.; Chengping, L. In vitro biofilm forming potential of Streptococcus suis isolated from human and swine in China. Braz. J. Microbiol. 2012, 43, 993-1004. [CrossRef] [PubMed]

32. Costerton, J.W. Introduction to biofilm. Int. J. Antimicrob. Agents 1999, 11, 217-221,237-239. [CrossRef]

33. Chuzeville, S.; Auger, J.P.; Dumesnil, A.; Roy, D.; Lacouture, S.; Fittipaldi, N.; Grenier, D.; Gottschalk, M. Serotype-specific role of antigen I/II in the initial steps of the pathogenesis of the infection caused by Streptococcus suis. Vet. Res. 2017, 48, 39. [CrossRef] [PubMed]

34. Brady, L.J.; Maddocks, S.E.; Larson, M.R.; Forsgren, N.; Persson, K.; Deivanayagam, C.C.; Jenkinson, H.F. The changing faces of Streptococcus antigen I/II polypeptide family adhesins. Mol. Microbiol. 2010, 77, 276-286. [CrossRef] [PubMed] 
35. Schreur, P.J.; Rebel, J.M.; Smits, M.A.; van Putten, J.P.; Smith, H.E. Differential activation of the Toll-like receptor 2/ 6 complex by lipoproteins of Streptococcus suis serotypes 2 and 9. Vet. Microbiol. 2010, 143, 363-370. [CrossRef] [PubMed]

36. Meijerink, M.; Ferrando, M.L.; Lammers, G.; Taverne, N.; Smith, H.E.; Wells, J.M. Immunomodulatory effects of Streptococcus suis capsule type on human dendritic cell responses, phagocytosis and intracellular survival. PLoS ONE 2012, 7, e35849. [CrossRef] [PubMed]

37. Melancon, D.; Grenier, D. Production and properties of bacteriocin-like inhibitory substances from the swine pathogen Streptococcus suis serotype 2. Appl. Environ. Microbiol. 2003, 69, 4482-4488. [CrossRef] [PubMed]

38. Li, Y.; Weinberger, D.M.; Thompson, C.M.; Trzciński, K.; Lipsitch, M. Surface charge of Streptococcus pneumoniae predicts serotype distribution. Infect. Immun. 2013, 81, 4519-4524. [CrossRef] [PubMed]

39. Hathaway, L.J.; Brugger, S.D.; Morand, B.; Bangert, M.; Rotzetter, J.U.; Hauser, C.; Graber, W.A.; Gore, S.; Kadioglu, A.; Mühlemann, K. Capsule type of Streptococcus pneumoniae determines growth phenotype. PLoS Pathog. 2012, 8, e1002574. [CrossRef] [PubMed]

40. Tobias, T.J.; Bouma, A.; Daemen, A.J.; Wagenaar, J.A.; Stegeman, A.; Klinkenberg, D. Association between transmission rate and disease severity for Actinobacillus pleuropneumoniae infection in pigs. Vet. Res. 2013, 44. [CrossRef] [PubMed]

41. Loera-Muro, A.; Jacques, M.; Avelar-González, F.J.; Labrie, J.; Tremblay, Y.D.; Oropeza-Navarro, R.; Guerrero-Barrera, A.L. Auxotrophic Actinobacillus pleurpneumoniae grows in multispecies biofilms without the need for nicotinamide-adenine dinucleotide (NAD) supplementation. BMC Microbiol. 2016, 16, 128. [CrossRef] [PubMed]

42. Wu, N.H.; Meng, F.; Seitz, M.; Valentin-Weigand, P.; Herrler, G. Sialic acid-dependent interactions between influenza viruses and Streptococcus suis affect the infection of porcine tracheal cells. J. Gen. Virol. 2015, 96, 2557-2568. [CrossRef] [PubMed]

43. De Greeff, A.; van Selm, S.; Buys, H.; Harders-Westerveen, J.F.; Tunjungputri, R.N.; de Mast, Q.; van der Ven, A.J.; Stockhofe-Zurwieden, N.; de Jonge, M.I.; Smith, H.E. Pneumococcal colonization and invasive disease studied in a porcine model. BMC Microbiol. 2016, 16, 102. [CrossRef] [PubMed]

44. De Greeff, A.; Wisselink, H.J.; de Bree, F.M.; Schultsz, C.; Baums, C.G.; Thi, H.N.; Stockhofe-Zurwieden, N.; Smith, H.E. Genetic diversity of Streptococcus suis isolates as determined by comparative genome hybridization. BMC Microbiol. 2011, 11, 161. [CrossRef] [PubMed]

45. Velthuis, A.G.; Bouma, A.; Katsma, W.E.; Nodelijk, G.; De Jong, M.C. Design and analysis of smallscale transmission experiments with animals. Epidemiol. Infect. 2007, 135, 202-217. [CrossRef] [PubMed]

46. Berthelot-Hérault, F.; Gottschalk, M.; Labbé, A.; Cariolet, R.; Kobisch, M. Experimental airborne transmission of Streptococcus suis capsular type 2 in pigs. Vet. Microbiol. 2001, 82, 69-80. [CrossRef]

47. Dekker, N.; Bouma, A.; Daemen, I.; Klinkenberg, D.; van Leengoed, L.; Wagenaar, J.A.; Stegeman, A. Effect of spatial separation of pigs on spread of Streptococcus suis serotype 9. PLoS ONE 2013, 8, e61339. [CrossRef] [PubMed]

48. Stegeman, A.; Van Nes, A.; de Jong, M.C.; Bolder, F.W. Assessment of the effectiveness of vaccination against pseudorabies in finishing pigs. Am. J. Vet. Res. 1995, 56, 573-578. [PubMed]

49. Bouma, A. Determination of the effectiveness of pseudorabies marker vaccines in experiments and field trials. Biologicals 2005, 33, 241-245. [CrossRef] [PubMed]

50. Baums, C.G.; Bruggemann, C.; Kock, C.; Beineke, A.; Waldmann, K.H.; Valentin-Weigand, P. Immunogenicity of an autogenous Streptococcus suis bacterin in preparturient sows and their piglets in relation to protection after weaning. Clin. Vaccine Immunol. 2010, 17, 1589-1597. [CrossRef] [PubMed]

51. Beineke, A.; Bennecke, K.; Neis, C.; Schröder, C.; Waldmann, K.H.; Baumgärtner, W.; Valentin-Weigand, P.; Baums, C.G. Comparative evaluation of virulence and pathology of Streptococcus suis serotypes 2 and 9 in experimentally infected growers. Vet. Microbiol. 2008, 128, 423-430. [CrossRef] [PubMed]

52. Dekker, C.N.; Bouma, A.; Daemen, A.J.; van Leengoed, L.A.; Jonker, F.H.; Wagenaar, J.A.; Stegeman, J.A. Homologous whole bacterin vaccination is not able to reduce Streptococcus suis serotype 9 strain 7997 transmission among pigs or colonization. Vaccine 2012, 30, 1379-1387. [CrossRef] [PubMed]

53. Wisselink, H.J.; Reek, F.H.; Vecht, U.; Stockhofe-Zurwieden, N.; Smits, M.A.; Smith, H.E. Detection of virulent strains of Streptococcus suis type 2 and highly virulent strains of Streptococcus suis type 1 in tonsillar specimens of pigs by PCR. Vet. Microbiol. 1999, 67, 143-157. [CrossRef] 
54. Devriese, L.A.; Ceyssens, K.; Hommez, J.; Kilpper-Balz, R.; Schleifer, K.H. Characteristics of different Streptococcus suis ecovars and description of a simplified identification method. Vet. Microbiol. 1991, 26, 141-150. [CrossRef]

55. Dekker, N.; Daemen, I.; Verstappen, K.; de Greeff, A.; Smith, H.; Duim, B. Simultaneous Quantification and Differentiation of Streptococcus suis Serotypes 2 and 9 by Quantitative Real-Time PCR, Evaluated in Tonsillar and Nasal Samples of Pigs. Pathogens 2016, 5, 46. [CrossRef] [PubMed]

56. Tobias, T.J.; Bouma, A.; Klinkenberg, D.; Daemen, A.J.; Stegeman, J.A.; Wagenaar, J.A.; Duim, B. Detection of Actinobacillus pleuropneumoniae in pigs by real-time quantitative PCR for the apxIVA gene. Vet. J. 2012, 193, 557-560. [CrossRef] [PubMed]

57. Smith, H.E.; Damman, M.; van der Velde, J.; Wagenaar, F.; Wisselink, H.J.; Stockhofe-Zurwieden, N.; Smits, M.A. Identification and characterization of the cps locus of Streptococcus suis serotype 2: The capsule protects against phagocytosis and is an important virulence factor. Infect. Immun. 1999, 67, 1750-1756. [PubMed]

58. Dohoo, I.; Martin, W.; Stryhn, H. Veterinary Epidemiologic Research, 2nd ed.; AVC Inc.: Charlottetown, PE, Canada, 2007; 706p, ISBN 978-0-919013-41-4.

59. Pinheiro, J.; Bates, D.; DebRoy, S.; Sarkar, D.; R Development Core Team. Nlme: Linear and Nonlinear Mixed Effects Models; R Package Version 3.1-103; The R Foundation: Vienna, Austria, 2012.

60. Becker, N.G. Analysis of Infectious Disease Data, 1st ed.; Chapman and Hall: London, UK, 1989; pp. $139-174$. ISBN 978-0412309908.

61. Kroese, A.H.; De Jong, M.C.M. Design and analysis of transmission experiments. In Proceedings of the Meeting for the Society for Veterinary Epidemiology and Preventive Medicine, Noordwijkerhout, The Netherlands, 28-30 March 2001; pp. xxi-xxxvii.

62. Velthuis, A.G.; De Jong, M.C.; Kamp, E.M.; Stockhofe, N.; Verheijden, J.H. Design and analysis of an Actinobacillus pleuropneumoniae transmission experiment. Prev. Vet. Med. 2003, 60, 53-68. [CrossRef]

63. Mccullagh, P.; Nelder, J.A. Generalized Linear Models, 2nd ed.; Chapman and Hall: London, UK, 1989; 506p, ISBN 978-0412317606.

(C) 2017 by the authors. Licensee MDPI, Basel, Switzerland. This article is an open access article distributed under the terms and conditions of the Creative Commons Attribution (CC BY) license (http:/ / creativecommons.org/licenses/by/4.0/). 